\title{
THE GREAT BURST OF MAY 23, 1967*
}

\author{
John P. Castelli, Jules Aarons and \\ Glen A. Michael \\ (Air Force Cambridge Research \\ Laboratories, Bedford, Mass., U.S.A.) \\ (Air Weather Service, Ent AFB, \\ Colo., U.S.A.)
}

\begin{abstract}
A BSTRACT
One of the largest radio bursts on record took place on May 23, 1967. Peak flux densities ranged between 23000 flux units $\left(10^{-22} \mathrm{wm}^{-2} \mathrm{~Hz}^{-1}\right)$ at $8800 \mathrm{MHz}$ to about 370000 units at $606 \mathrm{MHz}$. In addition to the high-accuracy measurements of the peak flux densities at $606,1415,2695,4995$, and $8800 \mathrm{MHz}$, sweep frequency observations from 19-39 MHz show Type-IV emission with Type-II bursts occurring during the Type-IV continuum. The associated flare was clearly visible in white light. In reviewing and compiling microwave-radio data recorded during earlier white-light flares, it was found that all but one of eight events listed by Svestka (1966) had high microwave flux densities associated with them. In comparing radio-burst intensity with optical flare importance for the series of three flares between 1809 and 2150 UT on May 23, there is only moderate agreement. The first radio burst was small; the third was by far the largest, while the second flare had the highest optical classification. The flux densities of the third burst may have been the highest ever recorded in the decimeter portion of the radio spectrum and amongst the largest four in the $8800 \mathrm{MHz}$ region. It is suggested that the details of the development of the flare might be followed on radio flux-density plots to determine detailed correlation with particle events in space and with terrestrial effects.
\end{abstract}

* Presented by J. P. Castelli.

Kiepenheuer (ed.), Structure and Development of Solar Active Regions. 601. I.A.U. 